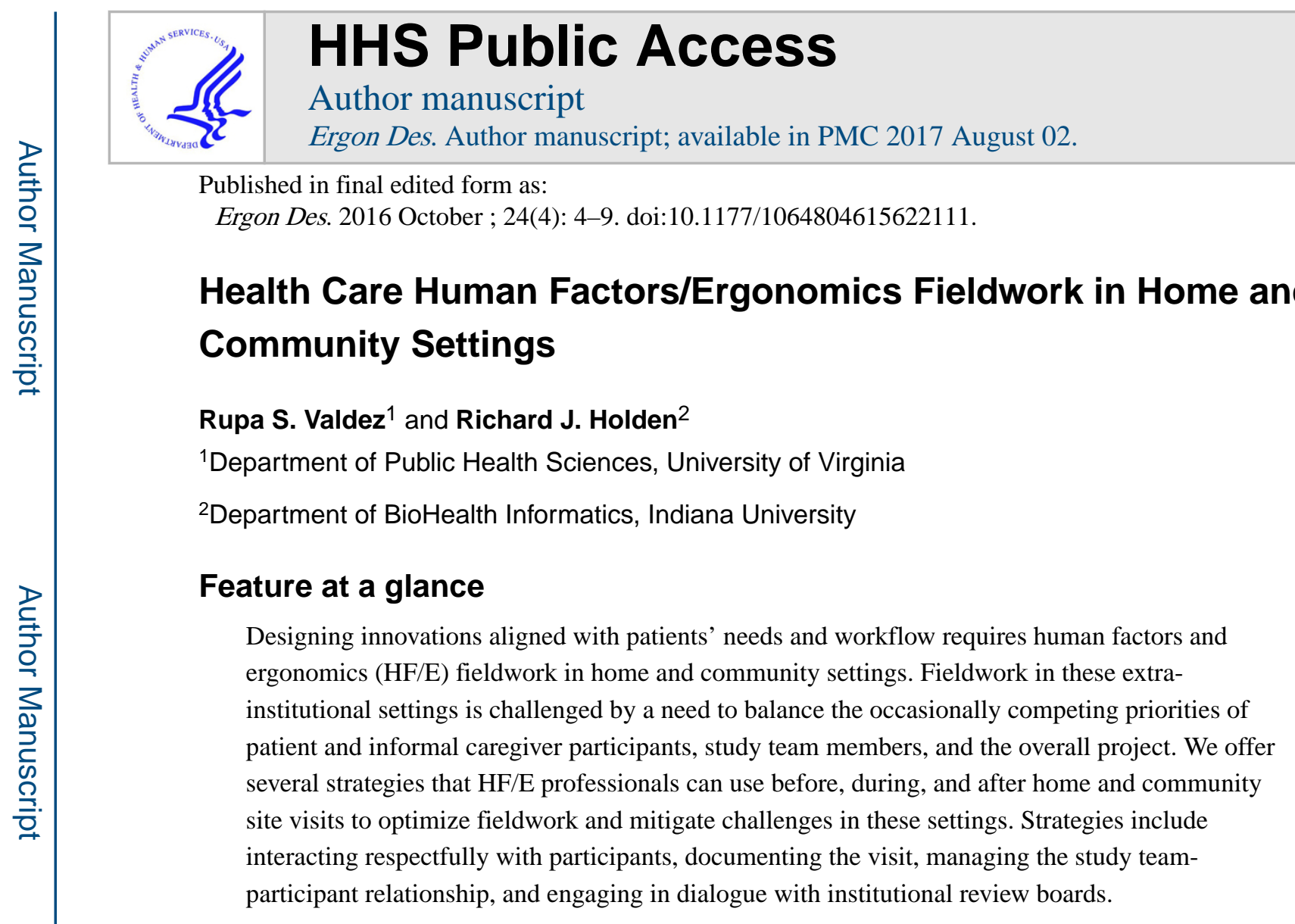

\title{
Keywords
}

Fieldwork; patients; informal caregivers; extra-institutional settings; work systems; health information technology

\section{Short Feature}

\section{Health care HF/E: moving into the home and community}

Human factors/ergonomics $(\mathrm{HF} / \mathrm{E})$ has contributed to improving health care for over half a century (Chapanis \& Safrin, 1960) and is increasingly recognized as a key driver for advances in health care quality and safety (Institute of Medicine, 2011; National Academy of Engineering \& Institute Medicine, 2005). Meaningful contributions in the future will be stimulated by two paradigm shifts, which are transforming the targets and settings of health care $\mathrm{HF} / \mathrm{E}$ practice and research. The first shift concerns the unit of analysis. In $\mathrm{HF} / \mathrm{E}$, the focus has extended from physical to cognitive to sociotechnical systems (Holden, Rivera, \& Carayon, 2015). In health care, the focus has similarly progressed from biomedical to psychological to systems approaches (Valdez, Holden, Novak, \& Veinot, 2015).

Consequently, it is increasingly recognized that health care HF/E intervention design, whether technological or programmatic, must account for physical, organizational, and social environments that comprise the larger system context (Waterson, 2009). The second paradigm shift concerns the scope of health care. While HF/E practice within health care originated within institutional settings such as hospitals and clinics (Chapanis \& Safrin, 1960), it is increasingly acknowledged as also encompassing home and community settings (e.g., self-regulating blood glucose, managing health information) (Holden, Schubert, \& 
Mickelson, 2015; Moen \& Brennan, 2005; Zayas-Caban \& Valdez, 2011). This transition is the result of multiple trends, including increased fragmentation of care, insurance based pressures for earlier discharge, proliferation of health information technology, and cultural shifts emphasizing patient engagement and shared decision-making (Brennan, Downs, and Casper, 2010; Carman et al., 2013; Gruman et al., 2010). To be responsive to this shift of scope, HF/E interventions must not only consider professionals (e.g., physicians, nurses, therapists) but also patients, family members, friends, and others in their community. The intersection of these two paradigm shifts implies that a new frontier for HF/E in health care is a sociotechnical systems approach that considers health care as a system including the home and community (Figure 1). Such an approach is relevant for studying and developing interventions to address a range of phenomena including transitions of care, chronic illness management, care coordination, and health information technology (Carayon et al., 2013).

Existing HF/E tools and concepts relevant to a sociotechnical systems approach require adaptation to be effectively applied to home and community settings. In comparison to hospital and clinic environments, patients' homes and communities are highly personal spaces in which health care activities are enmeshed with many other activities of daily living (Corbin \& Strauss, 1985) (Figure 2). Moreover, unlike health care professionals, patients and the individuals that support them are not typically paid to engage in health-related work. Work system models have been developed that specifically attend to health-related work in extra-institutional settings (Holden et al., 2013; National Research Council, 2011). Similarly, efforts are underway to translate the concept of workload for the patient context (NathanRoberts, Holden, Yin, \& Valdez, 2015). In addition to theoretical and methodological considerations, home and community environments raise unique challenges for initiating and conducting fieldwork (Furniss et al., 2014; Holden, Scott, Hoonakker, Hundt, \& Carayon, 2014).

The HF/E community is amassing experience within home and community environments through projects spanning health care phenomena (e.g., care coordination, self-monitoring, personal health information management), patient diagnoses (e.g., chronic obstructive pulmonary disease, hypertension, Type 2 diabetes), and designs (e.g., qualitative inquiry, clinical trials). These experiences illuminate the challenges and competing priorities that must be managed during fieldwork. Moreover, the lessons learned inform best practices for health care HF/E professionals to follow when interacting in this space.

\section{Challenges and competing priorities: the community, the study team, and the project}

There are multiple stakeholders whose priorities must be adequately addressed to successfully complete fieldwork in the home and community. The three primary stakeholders are: 1) community members, or the patients and informal caregivers whose activities and environments are the focus of inquiry, 2) study team members, or the HF/E professionals who are conducting the investigation, and 3) the project, which although not an independent stakeholder, has specific goals associated with its integrity and may be represented by a client or funder. Figure 3 details the priorities of each stakeholder. The challenge is balancing these priorities, which may conflict. Two brief examples are provided below: 
1. Javier leads a new project to develop an app for asthma management at home. The project has a short turnaround time; thus, the team has limited time to devote to fieldwork. During a visit with a key community partner, Javier is taken by surprise when he discovers the individual has low literacy and asks for assistance with using the app. Javier is concerned about the amount of time this interaction will take, although he realizes that obtaining data from individuals with low literacy may provide unique insights, improving his product's quality.

2. Rachel investigates how social and physical environments impact the effectiveness of care coordination (i.e., organization of patient care activities and information sharing among all individuals involved in a patient's care). Her methods include interviews and still photography of the physical environment. During a home visit, a participant offers to show the challenge of storing medical equipment in a basement closet. Rachel is unsure how to proceed. The project would benefit from the photographs and Rachel is wary of offending or diminishing the participant's trust, but feels that venturing into a space far from an exit compromises her personal safety.

These cases illustrate only some of the challenges encountered in fieldwork. Many other examples are presented by Holden and colleagues (2015), who developed a framework of challenges encountered in home and community based health care HF/E fieldwork, including difficulties gaining trust from participants, problems interacting with sick or impaired patients, confidentiality and compensation challenges, and questions of data quality (Figure 4).

\section{Best practices: strategies for health care HF/E fieldwork in the home and community}

To address these challenges and competing priorities, best practices for health care $\mathrm{HF} / \mathrm{E}$ fieldwork in the home and community should be used before, during, and after a site visit. Prior to visiting a participant in an extra-institutional setting, assess potential challenges related to the legal and ethical implications of their interaction with human subjects, develop contingency plans, and obtain the necessary approval from an institutional human subjects authority. Establish contact with the participant to plan the logistical aspects of the visit. In collaboration with other members of the study team, determine how situations compromising safety and miscommunication would be addressed. During the site visit, demonstrate respect for the participant while simultaneously protecting personal safety and staying within the scope of $\mathrm{HF} / \mathrm{E}$ expertise. Also, be alert and flexible in responding to unexpected situations. After leaving the site visit, immediately document the experience, paying particular attention to unusual events and unique insights. Finally, before formally ending a relationship with a participant, extend the interactions as necessary to complete the study rigorously. Figure 5 provides a checklist of specific strategies that may be implemented when conducting health care HF/E fieldwork in the home and community.

Examples of strategies that could be used to address Javier and Rachel's challenges are provided below:

1. Javier may have been better prepared for a low-literacy participant if he had contacted the participant prior to the site visit and asked about special needs 
requiring accommodation. With this information, he could have obtained permission from his institution's human subjects authority to use an oral consent process and to provide assistance with app usage. Even if he had not followed the recommendations above, with an established protocol for managing unexpected situations, Javier would have been able to comfortably ask the participant to reschedule the meeting to give himself time to address these matters.

2. Rachel would have been better prepared to handle the invitation to visit a participant's basement if she were accompanied by another team member and had informed a third team member of her whereabouts. With two people at the site visit and a communication plan for relaying safety concerns, Rachel would have been more comfortable taking the requested photographs. In her fieldwork protocol, Rachel should also have committed to maintaining constant access to an exit. Despite these precautions, Rachel may still have had concerns about entering the basement, in which case she should have prepared phrases to respectfully decline this or other discomforting offers. For example, Rachel could have said, "Your example would be really useful for the study, but unfortunately I am running low on time today. Could we perhaps schedule another time to take the photograph?" or, "My supervisor does not allow me to enter people's basements, out of concern for safety."

The strategies we have recommended are grounded in our experience conducting health care $\mathrm{HF} / \mathrm{E}$ fieldwork in home and community settings and the guidance provided to us by the institutional review boards at the institutions with which we have been affiliated. For individuals whose activities in the home and community are overseen by an IRB, we recommend having a discussion about the specific protocol you intend to implement. In our own experiences we have encountered conflicting advice about the types of information that may be requested from or about potential participants prior to informed consent, the degree to which an interaction must be scripted, and how to determine study eligibility for individuals whose roles overlap (e.g., patient and caregiver). Building flexibility into the protocol is also advisable. For example, in Rachel's scenario the participant may have asked if he could take the picture and send it to her securely. If this form of data collection was written into the protocol, Rachel could have obtained the photographs without compromising her safety or inconveniencing the participant with a second visit.

\section{Conclusion}

HF/E professionals working in health care are committed to designing innovations aligned with patients' needs and preferences. Laboratory-based assessments are limited in that they primarily facilitate an understanding of interactions between the user, the task, and the technology. Designing systems that are also responsive to patients' social, organizational, and physical environments requires assessing needs and preferences in the home and community, as well. However, fieldwork in extra-institutional settings is challenging and requires balancing the priorities of multiple stakeholders. By articulating strategies to address these challenges and competing priorities, we aim to provide a foundation for best practices for health care HF/E fieldwork in the home and community. Future work is needed to address the many challenges that arise from conducting fieldwork in this new domain, 
such as translating an abundance of field data into concrete design recommendations (Valdez, Holden, Novak, and Veinot, 2015). and adapting HF/E paradigms for systemsoriented assessments of home and community-based health care.

\section{Acknowledgments}

RSV was supported by grants R36 HS 018809-01 and 1 R03 HS022930-01 from the Agency for Healthcare Research and Quality (AHRQ). RJH is supported by grant K01AG044439 from the National Institute on Aging (NIA) of the US National Institutes of Health (NIH). The content is solely the responsibility of the authors and does not necessarily represent the official views of AHRQ or NIH. Additionally, the authors would like to thank Patti Brennan, Pascale Carayon, Peter Hoonakker, Ann Hundt, Jenna Marquard, Enid Montague, Calvin Or, Dan NathanRoberts, Teresa Zayas-Caban, and all community partners and study participants.

\section{Biographies}

Rupa Valdez is assistant professor of biomedical informatics at the University of Virginia. She received her $\mathrm{PhD}$ in industrial and systems engineering from the University of Wisconsin-Madison in 2012. Her research draws on methods from human factors, medical informatics, population health, and cultural anthropology to conduct home and community fieldwork for the purpose of guiding and evaluating consumer health information technology design. She may be reached at rupa.valdez@virginia.edu.

Richard Holden is assistant professor of BioHealth Informatics at the Indiana University School of Informatics and Computing, Indianapolis. He received a joint $\mathrm{PhD}$ in industrial engineering and psychology from the University of Wisconsin-Madison in 2009. His research applies human factors to study and improve the work performance of patients, informal caregivers, and clinicians. He has investigated multiple healthcare interventions, including information technology, team-based care, and lean process redesign. He may be reached at rjholden@iupui.edu.

\section{References}

Brennan PF, Downs S, Casper G. Project HealthDesign: rethinking the power and potential of personal health records. Journal of Biomedical Informatics. 2010; 43(5 Suppl):S3-5. [PubMed: 20937482]

Carayon P, Karsh BT, Gurses A, Holden R, Hoonakker P, Hundt AS, Wetterneck TB. Macroergonomics in healthcare quality and patient safety. Review of Human Factors and Ergonomics. 2013; 8(1):4-54. [PubMed: 24729777]

Carman KL, Dardess P, Maurer M, Sofaer S, Adams K, Bechtel C, Sweeney J. Patient and family engagement: a framework for understanding the elements and developing interventions and policies. Health Affairs. 2013; 32(2):223-231. [PubMed: 23381514]

Chapanis A, Safrin MA. Of misses and medicines. Journal of Chronic Diseases. 1960; 12(4):403-408. [PubMed: 13692372]

Corbin J, Strauss AL. Managing chronic illness at home: three lines of work. Qualitative Sociology. 1985; 8(3):224-247.

Furniss D, O'Kane A, Randell R, Taneva S, Mentis H, Blandford A. Fieldwork for Healthcare: Case Studies Investigating Human Factors in Computing Systems. Morgan \& Claypool. 2014

Gruman J, Rovner MH, French ME, Jeffress D, Sofaer S, Shaller D, Prager DJ. From patient education to patient engagement: implications for the field of patient education. Patient Education and Counseling. 2010; 78(3):350-356. [PubMed: 20202780]

Holden RJ, Rivera AJ, Carayon P. Occupational macroergonomics: principles, scope, value, and methods. IIE Transactions on Occupational Ergonomics and Human Factors. 2015 
Holden RJ, Scott AMM, Hoonakker PLT, Hundt AS, Carayon P. Data collection challenges in community settings: insights from two field studies of patients with chronic disease. Quality of Life Research. 2014; 24(5):1043-55. [PubMed: 25154464]

Holden RJ, Schubert CC, Mickelson RS. The patient work system: An analysis of self-care performance barriers among elderly heart failure patients and their informal caregivers. Applied Ergonomics. 2015; 47:133-150. [PubMed: 25479983]

Holden RJ, Carayon P, Gurses AP, Hoonakker P, Hundt AS, Ozok AA, Rivera-Rodriguez AJ. SEIPS 2.0. A human factors framework for studying and improving the work of healthcare professionals and patients. Ergonomics. 2013; 56(11):1669-1686. [PubMed: 24088063]

Institute of Medicine. Health IT and Patient Safety: Building Safer Systems for Better Care. 2011

Moen A, Brennan PF. Health@Home: the work of health information management in the household (HIMH): implications for consumer health informatics (CHI) innovations. Journal of the American Medical Informatics Association. 2005; 12(6):648-656. [PubMed: 16049230]

Nathan-Roberts, D., Holden, RJ., Yin, S., Valdez, RS. Examining patient work: the when, and how much of self-care. Paper to be presented at the 19th Triennial Congress of the International Ergonomics Association Melbourne; Australia. (in press)

National Academy of Engineering, \& Institute of Medicine. Building a Better Delivery System: A New Engineering/Health Care Partnership. 2005

National Research Council. Health Care Comes Home: The Human Factors. Washington, D.C.: National Academies Press; 2011.

Valdez RS, Holden RJ, Novak LL, Veinot TC. Transforming consumer health informatics through a patient work framework: connecting patients to context. Journal of the American Medical Informatics Association. 2015; 22(1):2-10. [PubMed: 25125685]

Waterson P. A critical review of the systems approach within patient safety research. Ergonomics. 2009; 52(10):1185-1195. [PubMed: 19787499]

Zayas-Caban, T., Valdez, RS. Human factors in home care. In: Carayon, P., editor. Handbook of Human Factors and Ergonomics in Health Care and Patient Safety. Mahwah, NJ: Lawrence Erlbaum Associates; 2011. 


\section{A new frontier for health care human factors/ergonomics}

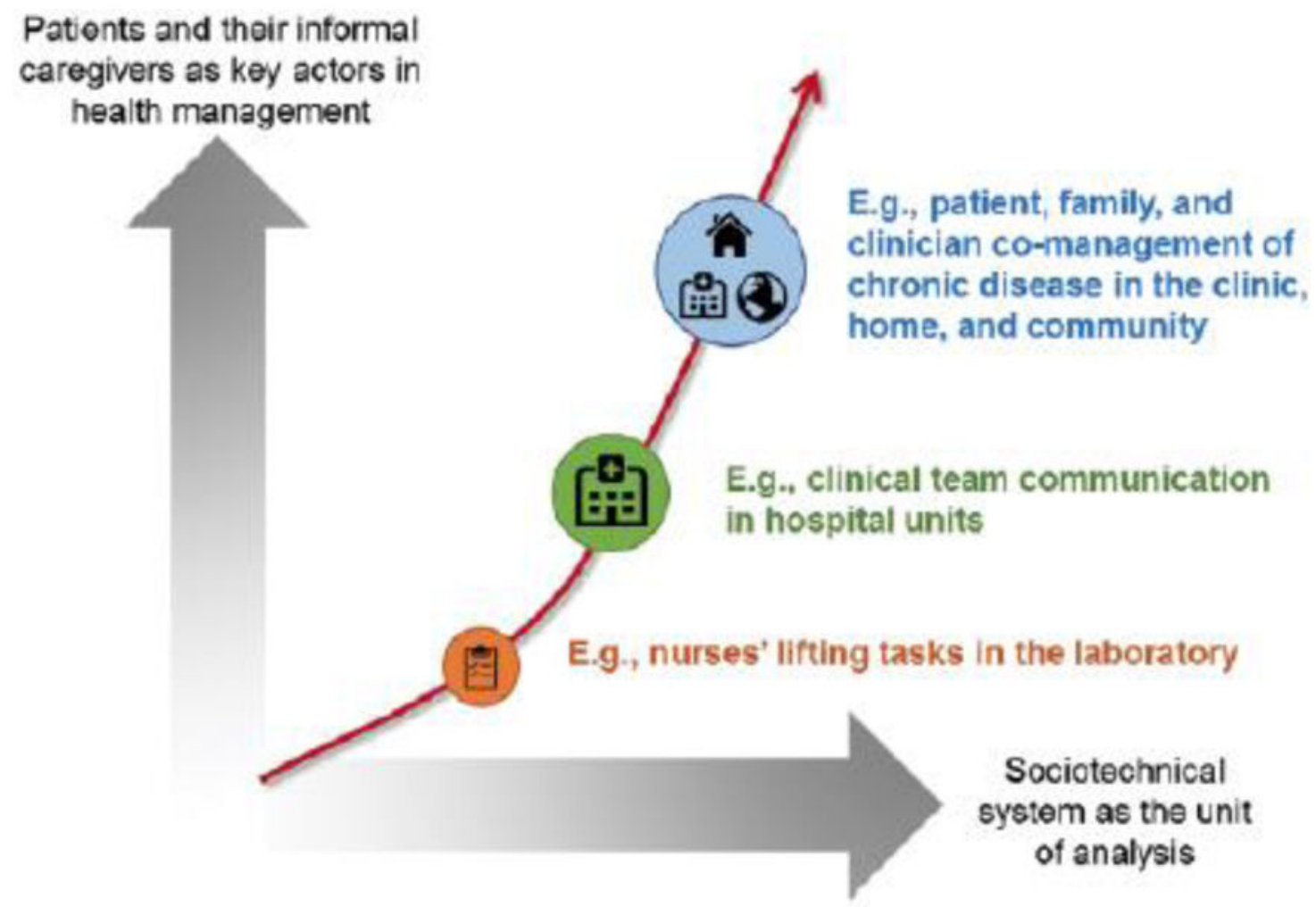

Figure 1.

Progression of human factors/ergonomics research in health care. 
Patient home environments are both complex and personal. Other aspects of the home might include pets, allergens, firearms, family members, and lighting or noise issues.
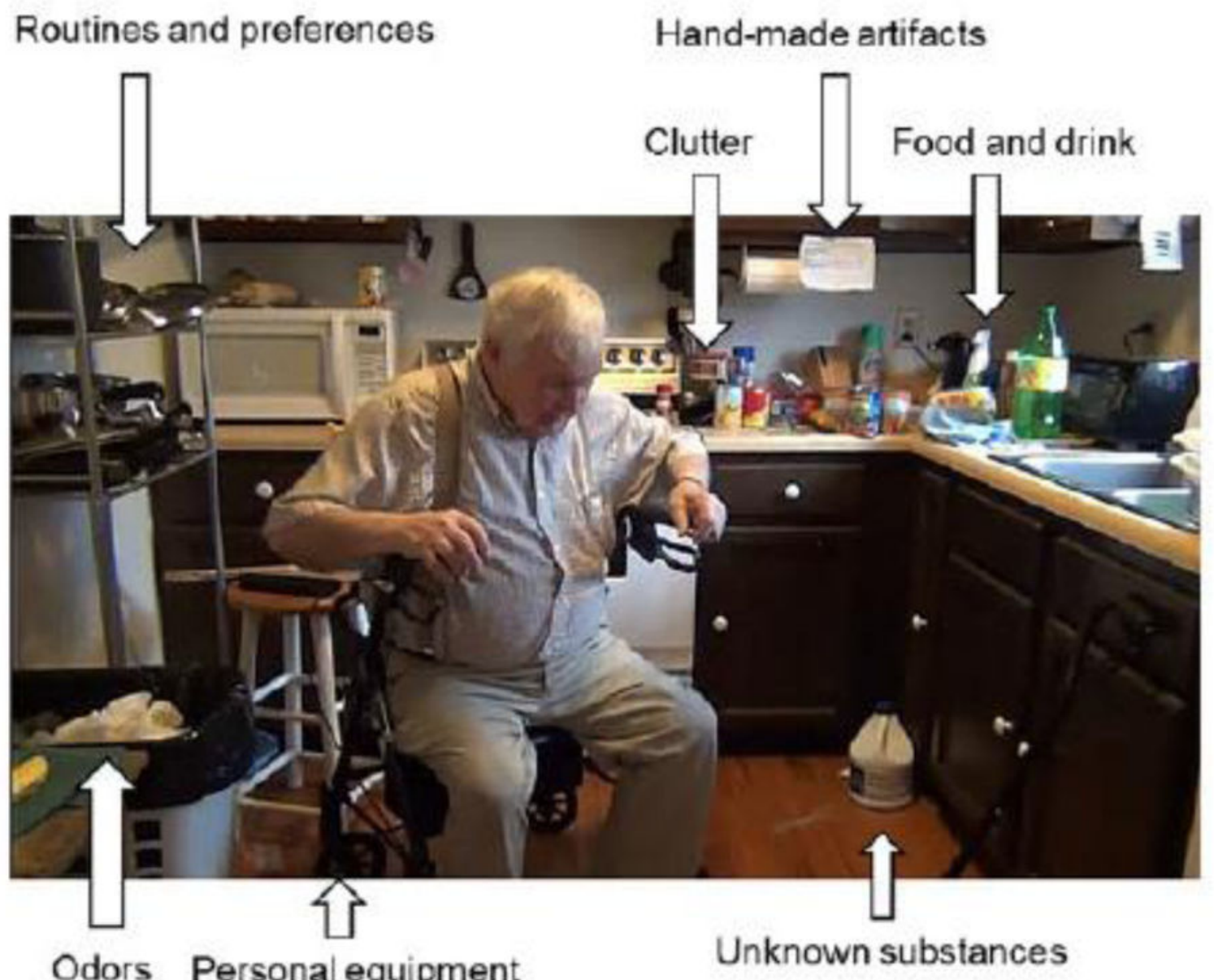

Figure 2.

Characteristics of patients' home environments. 


\section{The priorities of stakeholders in home and community based HF/E fieldwork}

\begin{tabular}{|c|c|}
\hline $\begin{array}{l}\text { Community Members } \\
\text { - Protect privacy of home and commun ity } \\
\text { cnvironments } \\
\text { - Project personal relationships that may be } \\
\text { implicated in the project scope } \\
\text { - Obtain adequate and timely compensation for } \\
\text { participation } \\
\text { - Mainta in ability to participate, regardless of } \\
\text { special considerations (e.g., physical/cognitive } \\
\text { limitations, language barriers) } \\
\text { - Obtain information and services beyond } \\
\text { the scope of the project (health } \\
\text { information, legal advice, home } \\
\text { maintenance, social services) } \\
\text { - Balance project participation with other } \\
\text { life demands } \\
\text { - Establish appropriate role for data collector } \\
\text { in the home/community space } \\
\text { - Provide socially desirable responses } \\
\text { - Maintain new strategies gleaned from } \\
\text { participation } \\
\text { - Minimize safety risks } \\
\text { - Share past experiences or present their } \\
\text { opinioins with an interested party. }\end{array}$ & $\begin{array}{l}\text { Study Team } \\
\text { - Minimize the time required to collect data } \\
\text { - Minimize safety risks } \\
\text { - Minimize discomfort } \\
\text { - Meet professional obligations (e.g. } \\
\text { documenting reportable events) } \\
\text { - Build relationship and establish trust with } \\
\text { participants } \\
\text { - Project reputation of the team and its } \\
\text { institutions }\end{array}$ \\
\hline
\end{tabular}

Figure 3.

The priorities of stakeholders in home- and community-based human factors/ergonomics fieldwork. 


\section{Challenges associated with fieldwork in the home and community environment (Adapted from Holden et al., 2015)}

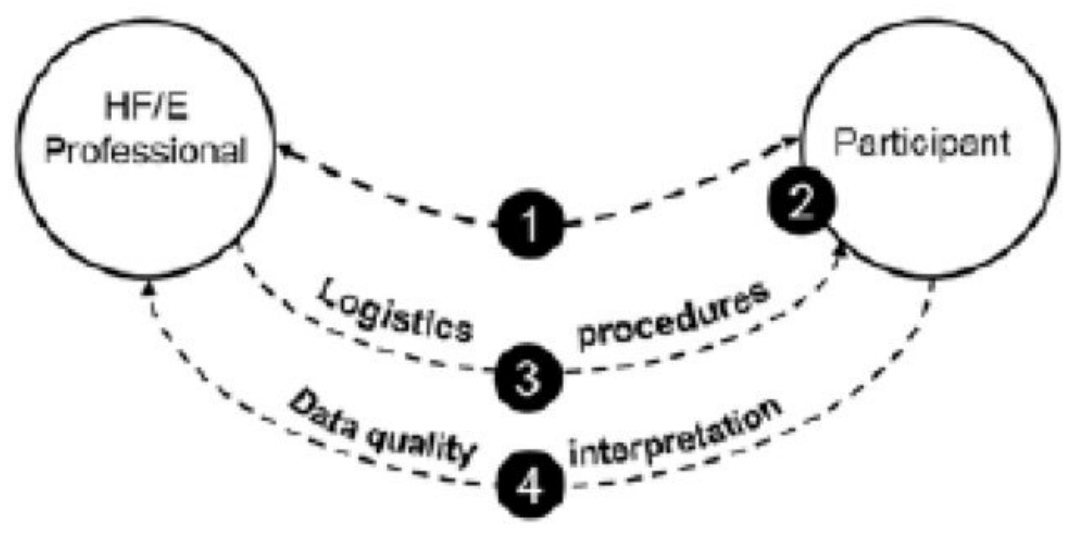

\section{Challenges of prolessional-partieipant partnership}

- HF/E professionalparticipant differences in priorites

- Mistrust and misunderstanding of HF/E professionals

- Differences in language, perspective, personal norms

\section{Challonges of participant characteristics}

- Participants' competing lise and health demands

- Psychosocial, cognitive, pereeptuallimitations

\section{Challenges of researeh logistics/procedures}

- Patient ident fication and recruirment

- Logistical iscues

- Maintaining participant privacy, confidentiality

- Conflicts with compensation

\section{Challongos of data quality and intorpretation}

- Questions of data quality interpretation, and integration of dats

Figure 4.

Challenges associated with fieldwork in the home and community environment. [Adapted from Holden, Scott, Hoonakker, Hundt, \& Carayon, 2015] 


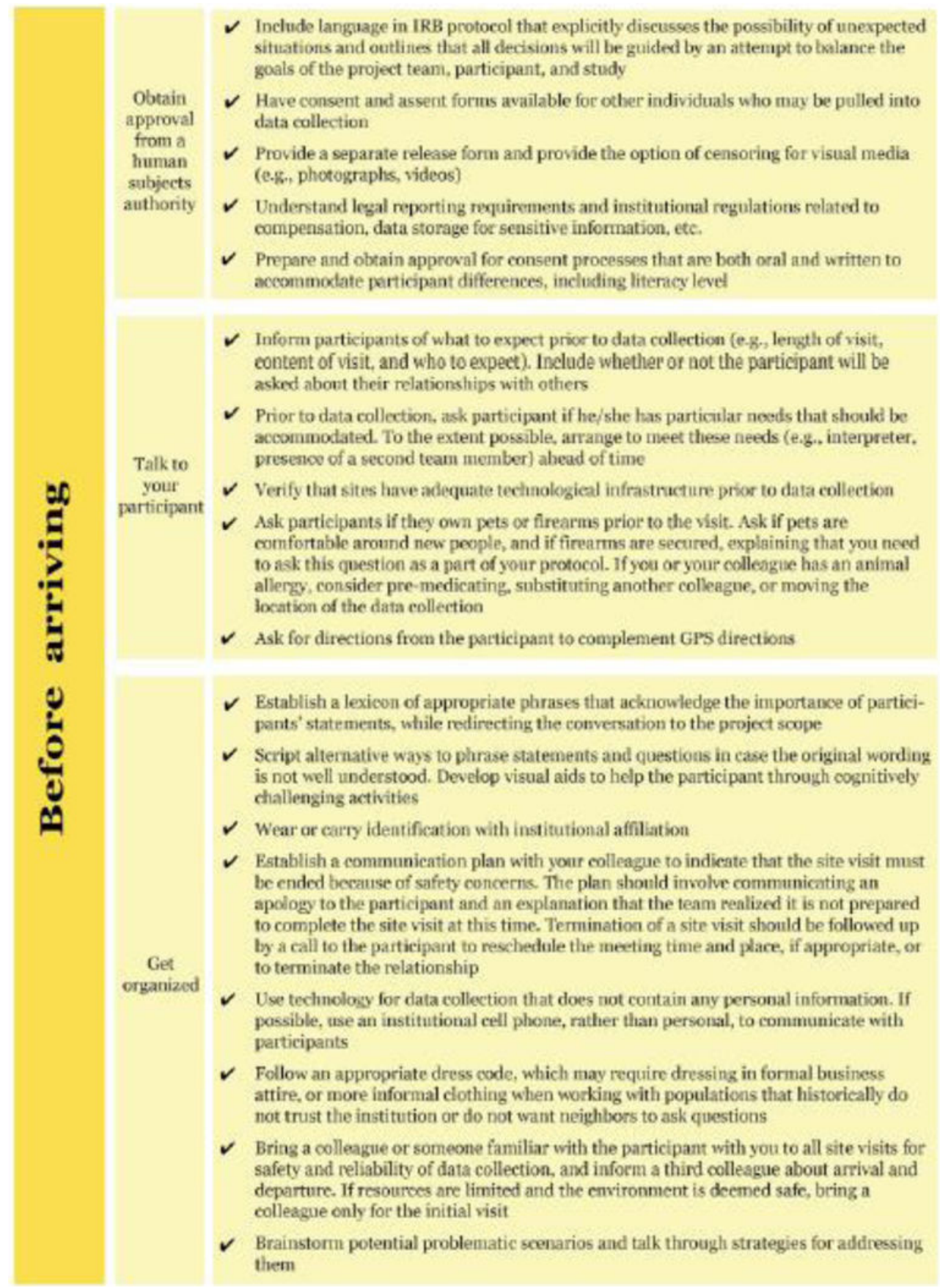

Ergon Des. Author manuscript; available in PMC 2017 August 02. 

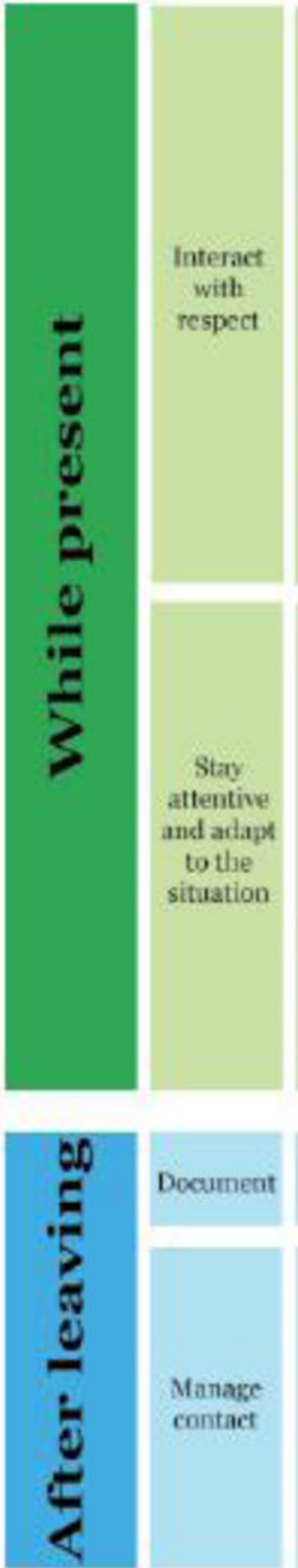

Document

Stay attentive and adaph to the situation

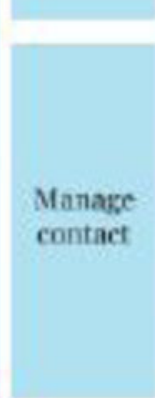

Acoegt requests to assist with minor tasks to facilitate relationship and trust building (e.g., check mail, download app/computer program) Respectfully decline offers of food and beverage by using phrases such as, "That looks wonderful, but I just ate."

$\checkmark$ If a participant expresses fatigue or the nood to address other responsibilities, respect this expression and reschedule a follow up visit

V Ask the participant if he/she would prefer to have materials in writing, or if they would prefer to have them read aloud.

$\checkmark$ Provide compensation immediately following data collection (i.e-, no waiting period) unless participants are willing to wait for compensation in a form requiring a waiting persod (e.g., check)

$\checkmark$ Respectfully, but directly respond to inquiries that are out of your scope of practice by stating that you cannot provide certain information or services.

$\checkmark$ Begin data collection with a statement acknowledging the diversity of experiences and importance of learning about the participant's unique perspective. Assure the participant that he/ she Is not being judged or graded in any way

To the extent possible, stay within communal spaces of the home (eg., kitchen, living room) and maintain an exit strategy

v Ask participant for guidance if an isste arises that he/she can help resolve (e.g. continuing the data collection with anothes member of the bousehold present, hunges, medical needs, unscheduled visitors or phone calls)

$\checkmark$ Signal an end to the interaction when there are about five minutrs remaining and use a closing gesture, such as providing the incentive and/or a business card with contact information if the participant should have any questions

$\checkmark$ Reflect the participant's vocabulary and demeanor, in terus of how they talk about liealth-related issues (e.g, blood pressure vs. hypertension) and level of energy

$\checkmark$ Be patient, open-minded and observant, adapting data collextion instruments when participants lead consersation in fruitual directions

Figure 5.

Health care human factors/ergonomics home and community fieldwork checklist. 